\title{
Hybrid ARQ Scheme with Antenna Permutation for MIMO Systems in Slow Fading Channels
}

\author{
Jianfeng Wang, Meizhen Tu, Kan Zheng, and Wenbo Wang \\ School of Telecommunication Engineering, \\ Beijing University of Posts \& Telecommunications, Beijing 100876, China \\ javenwang. bupt@gmail.com
}

\begin{abstract}
In this paper, an equivalent model for the hybrid automatic retransmission request (HARQ) multi-input multi-output (MIMO) systems with a proper combining scheme is first introduced. Based on this effective model, we present a simple technique, termed antenna permutation scheme (APS), which permutates the transmit antennas at each retransmission to improve the diversity gain from retransmissions in the slow fading environment. The theory analysis and simulation results demonstrate that the system with APS can achieve much better bit error performance.
\end{abstract}

Keywords: Hybrid ARQ, MIMO, V-BLAST, APS.

\section{Introduction}

Given a fixed bandwidth and power budget, an interesting approach to increase data rates is to use multiple antennas at both ends of a wireless link [1] [2] [3]. An attractive MIMO system to exploit this potential is the well-know Vertical Bell Labs Layered Space-Time (V-BLAST) architecture 3. On the other hand, reliable packet data service transmission should also be provided in the future communication systems. The use of hybrid automatic retransmission request (HARQ) is intended to ensure an extremely low packet error rate 4 for the packet communication.

Some combination of the V-BLAST system with the HARQ scheme to exploit the characteristic of the MIMO systems were proposed. H.Zheng [5] gave a information theoretic analysis on the BLAST system combined with HARQ with an equivalent structure while the combining algorithm on the receiver was not described. Onggosanusi 6] presented and compared two combining schemes based on the position of the HARQ cumulative combination before or after the V-BLAST detector. We find that the HARQ-MIMO system can be modelled as the equivalent structure [5] only if the pre-combing [6] scheme is applied.

To achieve more diversity gain from retransmissions in slow fading environment, we present and analyze a simple scheme, termed antenna permutation scheme (APS), which adapts spatial diversity gain into temporal diversity gain by permuting antennas at each retransmission and improves the performance efficiently. 
This paper is organized as follows. Section 2 gives the brief description of HARQ-MIMO system and the equivalent structure. Based on the structure, the antenna permutation scheme is described in details in Section 3 And in Section 4 the simulation results are presented and discussed. Finally, Section 5 gives the conclusion.

Notations: Throughout this paper, matrices and vectors are set in boldface. $(\cdot)^{T},(\cdot)^{H},(\cdot)^{+}$and $|\cdot|$ denote transpose, conjugate transpose, Moore-Penrose pseudo-inverse and determination of the matrix, respectively.

\section{HARQ-MIMO System}

Here we consider a V-BLAST scheme system with $N_{t}$ transmit antennas and $N_{r}$ receive antennas $\left(N_{r} \geq N_{t}\right)$. The received vector at the $i$-th transmission is written as:

$$
\mathbf{y}_{(i)}=\mathbf{H}_{(i)} \mathbf{x}+\mathbf{n}_{(i)}, i=1,2, \ldots, N
$$

where $\mathbf{x}=\left[x_{1} \cdots x_{N_{t}}\right]^{T}$ is the $N_{t} \times 1$ transmit symbols vector. $\mathbf{H}_{(i)}$ is the $N_{r} \times N_{t}$ channel matrix contains uncorrelated complex Gaussian fading gains with unit variance at the $i$-th retransmission. $\mathbf{n}_{(i)}=\left[n_{(i) 1} \cdots n_{(i) N_{r}}\right]$ represents the white Gaussian noise of variance $\sigma^{2}$ observed at the receive antennas. And $N$ denotes the number of transmissions for the same packet.

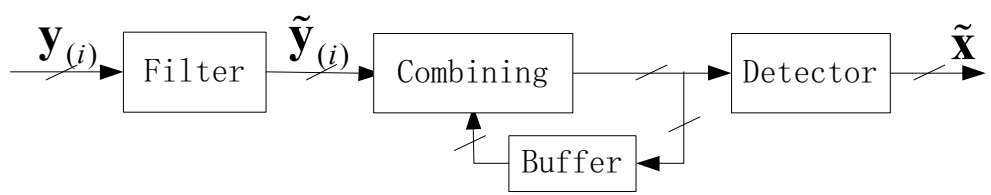

Fig. 1. Pre-combing scheme in the receiver of the HARQ-MIMO system

For the pre-combing receiver structure, as depicted in Figure 1 the received vector $\mathbf{y}_{(i)}$ and channel matrix $\mathbf{H}_{(i)}$ are first filtered with the matrix $\mathbf{H}_{(i)}^{H}$, yields $\tilde{\mathbf{y}}_{(i)}, \tilde{\mathbf{H}}_{(i)}$ respectively, i.e. $\tilde{\mathbf{y}}_{(i)}=\mathbf{H}_{(i)}^{H} \mathbf{y}_{(i)}$ and $\tilde{\mathbf{H}}_{(i)}=\mathbf{H}_{(i)}^{H} \mathbf{H}_{(i)}$ for further maximum ratio combing. Then the vectors after $N$ transmissions are cumulatively combined. After that, some detection algorithms used in the V-BLAST system can be applied. Here the linear zero-forcing (ZF) and minimum mean square error (MMSE) detectors are considered, then we can get:

$$
\begin{gathered}
\tilde{\mathbf{x}}_{Z F}=\left(\sum_{i=1}^{N} \tilde{\mathbf{H}}_{(i)}\right)^{-1} \sum_{i=1}^{N} \tilde{\mathbf{y}}_{(i)} \\
\tilde{\mathbf{x}}_{M M S E}=\left(\sum_{i=1}^{N} \tilde{\mathbf{H}}_{(i)}+\sigma^{2} \mathbf{I}_{N_{r}}\right)^{-1} \sum_{i=1}^{N} \tilde{\mathbf{y}}_{(i)}
\end{gathered}
$$




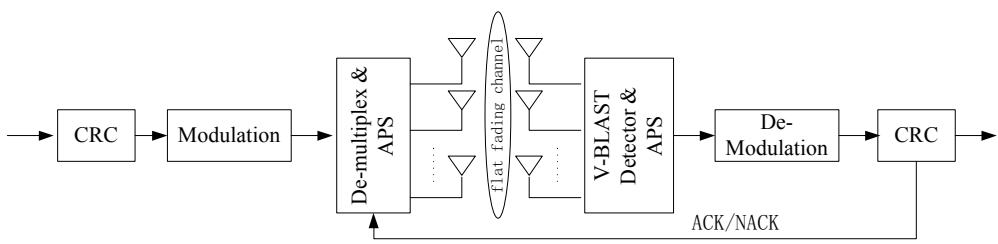

Fig. 2. HARQ-MIMO system block diagram with APS

which are not convenient to evaluate the performance directly for the cumulative sum. To facilitate our evaluation, we just adopt it to the matrix multiplication. According to the definition of $\tilde{\mathbf{y}}_{(i)}$ and $\tilde{\mathbf{H}}_{(i)}$, the equations can be rewritten as:

$$
\begin{gathered}
\tilde{\mathbf{x}}_{Z F}=\hat{\mathbf{H}}^{+} \hat{\mathbf{y}} \\
\tilde{\mathbf{x}}_{M M S E}=\left(\hat{\mathbf{H}}^{H} \hat{\mathbf{H}}+\sigma^{2} \mathbf{I}_{N_{r}}\right)^{-1} \hat{\mathbf{H}}^{H} \hat{\mathbf{y}}
\end{gathered}
$$

where $\hat{\mathbf{H}}=\left[\mathbf{H}_{(1)}^{T} \cdots \mathbf{H}_{(N)}^{T}\right]^{T}$ and $\hat{\mathbf{y}}=\left[\mathbf{y}_{(1)}^{T} \cdots \mathbf{y}_{(N)}^{T}\right]^{T}$. which indicate that the receiver of the HARQ-MIMO system with the pre-combining scheme can be modelled as a pure $\mathrm{V}$-BLAST system with $N \times N_{r}$ receiver antennas, which implies that the system performance can be analyzed on this simplified equivalent structure.

\section{Antenna Permutation Scheme}

Because the channels that each retransmission experience are almost fully correlated, i.e. $\mathbf{H}_{(i+1)} \doteq \mathbf{H}_{(i)}$ in the slow fading environment, the temporal diversity gain from the retransmissions is limited, which is the motivation to degrade the correlation between the retransmissions. Some approaches have been considered to make the channels upon the retransmissions uncorrelated, such as [6] where a precoder matrix is used on the transmitter side. Here we present a very simple scheme which permutes the transmit antennas on each retransmission to exploit the diversity gain.

\subsection{System Model}

The APS-HARQ-MIMO system block diagram is depicted in Fig 2 Here the packet is sent to be modulated after CRC check-sum added, after that the main stream is de-multiplexed into multiple sub-streams, i.e. multiple transmitter antennas, in a vertical way.

At the receiver, the sub-streams from each antenna are stored in the buffer and detected based on the some V-BLAST detector algorithm. Then the soft symbol are used to determine whether the packet is error or not by CRC check. 
Table 1. Antenna permutation for 2 and 4 transmit antennas

\begin{tabular}{c|c|c|c|c}
\hline Antennas & $1^{\text {st }}$ transmission & $2^{\text {nd }}$ transmission & $3^{\text {rd }}$ transmission & $\cdots$ \\
\hline 2 & $\{1,2\}$ & $\{2,1\}$ & $\{1,2\}$ & $\cdots$ \\
\hline 4 & $\{1,2,3,4\}$ & $\{3,4,1,2\}$ & $\{1,2,3,4\}$ & $\cdots$ \\
\hline
\end{tabular}

If the packet is declared error-free, ACK is sent back to the transmitter, the buffer is released and the next packet is sent. Otherwise, a NACK is sent for retransmission request and some antenna permutation is selected before the next retransmission. Then combined with the previous data in buffer, the packet is checked as before.

The permutation candidates are in a pre-determined table, the 2 and 4 transmit antennas permutation strategies are illustrated in the Table 1, where the permutation strategy of the 4 transmit antennas can be optimized.

\subsection{Theory Analysis}

In this subsection, the performance of the system with the APS is analyzed, assumed that there are two transmissions in the $(2,2)$ system, i.e. $N=2$, $N_{t}=N_{r}=2$. More antennas and transmissions can by extended straightly. The channel on each retransmission is assumed to be static, i.e. $\mathbf{H}_{(i)}=\mathbf{H}$, which can be conveniently represented by a matrix $\mathbf{H}=\left[\begin{array}{l}\mathbf{h}_{1} \mathbf{h}_{2}\end{array}\right]$.

Here we use the linear $\mathrm{ZF}$ detection as an illustration. The receive signal vector is multiplied with the filter matrix $\mathbf{G}_{Z F}$ :

$$
\mathbf{G}_{Z F}=\hat{\mathbf{H}}^{+}
$$

where $\hat{\mathbf{H}}$ is the equivalent channel matrix.

The estimation errors of the different layers correspond to the main diagonal elements of the error covariance matrix [7:

$$
\boldsymbol{\Phi}_{Z F}=E\left\{\left(\tilde{\mathbf{x}}_{Z F}-\mathbf{x}\right)\left(\tilde{\mathbf{x}}_{Z F}-\mathbf{x}\right)^{H}\right\}=\sigma_{n}^{2}\left(\hat{\mathbf{H}}^{H} \hat{\mathbf{H}}\right)^{-1}
$$

which equals the covariance matrix of the noise after the receive filter. So the average estimation errors from the diagonal elements is:

$$
M S E=\frac{1}{2} \sigma_{n}^{2} \operatorname{tr}\left(\left(\hat{\mathbf{H}}^{H} \hat{\mathbf{H}}\right)^{-1}\right)
$$

where $\operatorname{tr}($.$) denotes the trace of the matrix.$

For the system without APS, the channels of each retransmission are static. The equivalent channel matrix can be written as $\hat{\mathbf{H}}=\left[\mathbf{H}^{T} \mathbf{H}^{T}\right]^{T}$. So the average error:

$$
M S E_{w o}=\frac{1}{2} \sigma_{n}^{2} \operatorname{tr}\left(\left(\left[\mathbf{H}^{H} \mathbf{H}^{H}\right]\left[\begin{array}{l}
\mathbf{H} \\
\mathbf{H}
\end{array}\right]\right)^{-1}\right)=\frac{\sigma_{n}^{2}}{4|\mathbf{H}|^{2}}\left(\left\|\mathbf{h}_{1}\right\|_{F}^{2}+\left\|\mathbf{h}_{2}\right\|_{F}^{2}\right)
$$

where $\|\cdot\|_{F}$ denotes the Frobenius norm. 
Using permutation on the transmit antennas, the channel can be written equivalently as $\mathbf{H}=\left[\mathbf{h}_{2} \mathbf{h}_{1}\right]$. To represent the permutation operation, a permutation matrix $\mathbf{J}=\left[\begin{array}{ll}0 & 1 \\ 1 & 0\end{array}\right]$ is introduced. Then the equivalent channel matrix can be written as $\hat{\mathbf{H}}=\left[\mathbf{H}^{T}(\mathbf{H J})^{T}\right]^{T}$. Note that JHJ denotes the permutation on the main diagonal elements of the matrix $\mathbf{H}$, that is useful for the evaluations below.

Based on the equation (7), the error covariance matrix with APS can be written as:

$M S E_{w}=\frac{1}{2} \sigma_{n}^{2} \operatorname{tr}\left(\left(\left[\mathbf{H}^{H} \mathbf{J H}^{H}\right]\left[\begin{array}{c}\mathbf{H} \\ \mathbf{H J}\end{array}\right]\right)^{-1}\right)=\frac{\sigma_{n}^{2}\left(\left\|\mathbf{h}_{1}\right\|_{F}^{2}+\left\|\mathbf{h}_{2}\right\|_{F}^{2}\right)}{\left(\left\|\mathbf{h}_{1}\right\|_{F}^{2}-\left\|\mathbf{h}_{2}\right\|_{F}^{2}\right)^{2}+4|\mathbf{H}|^{2}}$

which is much less than (9) for the non-positive $\left(\left\|\mathbf{h}_{1}\right\|_{F}^{2}-\left\|\mathbf{h}_{2}\right\|_{F}^{2}\right)^{2}$, which is related with the correlation between the transmit antennas. According to (10), we can find that the HARQ-MIMO system with APS has more diversity gain from retransmission by introducing spatial diversity into the temporal diversity in a simple way, and especially the less correlation between the spatial channel, the better performance can be achieved.

\section{Numerical Results}

In this section, we simulate the BER performance for the HARQ-MIMO system using QPSK modulation in the slow fading channel by simulations. The packet is transmitted twice on the channel, which is also assumed constant on each retransmission to evaluate and compare the combining gain. The APS on 2 and 4 antennas we used here is shown in the Table 1.

Within the $(2,2)$ V-BLAST system, simulation results are presented on the left Figure 3 shows. It depicts the average BER vs. SNR for $N=2,4$ scenarios. The result shows that in the original HARQ-MIMO system without APS , the
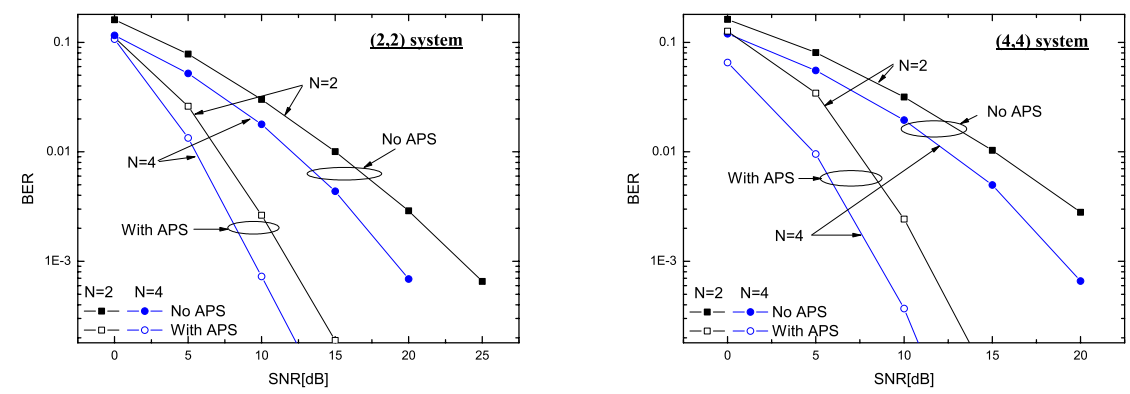

Fig. 3. Performance of the propose antenna permutation scheme in the V-BLAST system 
performance is about $3 \mathrm{~dB}$ better with the transmission number doubled, which is compliant with the analysis in the equation (9). Additionally, notice that the system with APS outperforms the original HARQ-MIMO system much better. At the BER of $10^{-2}$, the performance is about $7.5 \mathrm{~dB}$ far from the performance of the system without permutation.

The similar performance can be derived within the $(4,4)$ V-BLAST system as the right Figure 3 shows. It shows that the system with APS outperforms the original HARQ-MIMO about $6 \mathrm{~dB}$ at the BER of $10^{-2}$. We find that the system with APS can achieve much diversity degree by a very simple scheme for the situation that un-correlation between spatial channels.

\section{Conclusion}

In this paper, an effective and simple scheme, termed APS, is presented for the HARQ-MIMO system in the slow fading environment. To simplify the analysis, we first introduce and explain the rationality of the equivalent structure for the V-BLAST system with pre-combining scheme. Because of the limited retransmission gain, i.e. temporal diversity gain, we permutate the transmitter antennas on each retransmission which adopt the spatial un-correlation into the temporal domain. From the theory analysis and simulation results, the proposed scheme provides significant gain with very simple implementation complexity.

\section{References}

1. E.Telatar,"Capacity of Multi-antenna Gaussian Channels," European Transactions on Telecommunications, vol.10, pp. 585-595, November-December 2000.

2. G.J.Foschini," Layered space-time architechture for wireless communication in a fading environment when using multi-element antennas," Bell Labs Tech. J.,, pp.41-59, Aut 1996

3. P.W.Wolniansky, G.J.Foschini, G.D.Golden, R.A.Valenzuela,"V-BLAST: An Architechture for Realizing Very High Data Rates Over the Rich-Scattering Wireless Channel," Proc. ITG Conf. on Source and Channel Coding, Berlin, Germany, pp.41-59,January 2002.

4. S.Lin, D.J.Costello, M.J.Miller,"Automatic-repeat-request error-control schemes," IEEE Communications Magazine, vol.22, pp.5-17, December 1984.

5. H.Zheng,"Impact of Hybrid ARQ on BLAST Performance," CISS'01, vol.5, pp.32053209, May 2003.

6. E.N.Onggosanusi, A.G.Dabak, Y.H, and G.Jeong,"Hybrid ARQ Transmission and Combining for MIMO Systems," ICC'03, vol.5, pp.3205-3209, May 2003.

7. R.Bohnke, D.Wubben, V.Kuhn, and K.D.Kammeyer, "Reduced Complexity MMSE Detection for BLAST Architectures," GLOBECOM '03, IEEE, vol.4, pp.2258-2262, Dec. 2003. 